\title{
USOS Y COMPETENCIAS TIC EN ESTUDIANTES DE COMUNICACIÓN SOCIAL EN NORTE DE SANTANDER
}

\author{
USE AND COMPETENCES OF INFORMATION AND \\ COMMUNICATION TECHNOLOGY IN SOCIAL \\ COMMUNICATION STUDENTS OF NORTE THE \\ SANTANDER
}

Por

\section{Carolina García Pino ${ }^{1}$}

Universidad Francisco de Paula Santander - Cúcuta carolinagp@ufps.edu.co

Juan Camilo López Rojas ${ }^{2}$

Universidad Francisco de Paula Santander- Cúcuta juancamilolr@ufps.edu.co

\section{Luisa María Correa Ramírez}

Universidad Francisco de Paula Santander - Cúcuta luisamariacr@ufps.edu.co 
Resumen: El semillero de investigación en comunicación SEMINCO, de la Universidad Francisco de Paula Santander, se planteó la tarea de identificar los usos que dan los estudiantes de comunicación social a las herramientas tecnológicas y qué competencias éticas, investigativas y profesionales han desarrollado al finalizar su proceso formativo. Para ello, se tomó como muestra 105 estudiantes de último semestre de cuatro programas de comunicación social de Norte de Santander, abordando el uso de la tecnología para edición de audio, vídeo, texto y fotografía y para la creación de páginas web, blogs y redes sociales. Los resultados evidencian un bajo nivel de uso y conocimiento de la tecnología que requiere su quehacer profesional, especialmente en comunicación digital. El principal reto para la academia será promover una cultura de actualización permanente para lograr el aprendizaje autónomo y ofertar espacios para asegurar que sus egresados respondan a los requerimientos del mercado.

Palabras Clave: Usos TIC, competencias TIC, comunicador social.

Abstract: The research seed in communication SEMINCO of the Francisco de Paula Santander University, has proposed the task to identify the use that social communication student's give to technology tools and the ethical, investigative and professional competences developed in their career. To do so, there was taken a sample of 105 students that where in their last semester in four social communication programs in Norte the Santander, asking them about the use of technology to edit audio, video, text and photography, web site creation, blogs and social media. The findings show a low level of use and knowledge about the technology that required their professional work, especially in digital communication. The main challenge for the academy will be to promote a permanent updating culture to achieve the autonomy learning in their graduate to assure they respond to the market requirements.

Key Words: Use of information and communication technologies, competence, social communicator.

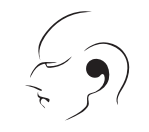

En la actualidad se exige a los profesionales de todas las áreas contar con competencias en el uso de la tecnología, que les permitan diferenciarse y aportar un valor agregado a las entidades laborales a las cuales aspiran, esto es especialmente relevante en el caso de los comunicadores sociales, quienes han visto en la web un nuevo campo laboral. 
Ese campo del saber se plantea como reto en la formación profesional de los comunicadores sociales, pues no sólo deben estar preparados para cualquier necesidad que pueda presentarse en su entorno laboral desde la comunicación, sino que - como parte de la sociedad digital-deben saber usar las TIC y contar con competencias idóneas en el uso de redes sociales, plataformas digitales, editores de contenido radial, visual y audiovisual, procesadores de texto, bases de datos, uso técnico de la cámara, gestión del trabajo en equipo, entre otras.

Arras, Torres y García-Valcárcel (2011) clasifican estas competencias en:

A) competencias básicas de alfabetización digital, que se relacionan con el uso de las TIC en las actividades del aula y las presentaciones, involucra la aplicación de herramientas digitales para obtener información y el uso y desarrollo de materiales utilizando diversas fuentes en línea;

B) competencias de aplicación, que están vinculadas con el uso de habilidades y conocimientos para crear y gestionar proyectos complejos, resolver problemas en situaciones del mundo real, colaborar con otros, y hacer uso de las redes de acceso a la información y a los expertos;

C) competencias éticas, cuando una persona entiende y demuestra el uso ético legal y responsable de las TIC (p. 5).

Así, se tiene que los futuros profesionales en el campo de la comunicación deben, en primer lugar, conocer las herramientas tecnológicas a su disposición para el ejercicio profesional, desarrollar habilidades en su uso y conocimientos que faciliten la producción mediática para múltiples formatos y plataformas y, finalmente, garantizar que tal uso se de en forma ética y responsable.

A partir de esta premisa surge la investigación Usos y competencias TIC en estudiantes de comunicación social en Norte de Santander, para describir qué herramientas tecnológicas conocen y usan los estudiantes de último semestre de los programas de comunicación social y qué competencias éticas, investigativas y profesionales han desarrollado en su formación profesional.

\section{Metodología}

La investigación de corte cuantitativo, tomó como población a los estudiantes de Comunicación Social de cuatro universidades de Norte de Santander, cursantes de séptimo y octavo semestre. Las universidades seleccionadas fueron la Universidad de Pamplona (seccionales Pamplona y Villa del Rosario) y La Universidad Francisco de Paula Santander (seccionales Cúcuta y Ocaña). Para acceder a los estudiantes se solicitó permiso a los directores de cada programa para aplicar la encuesta en una 
clase del penúltimo semestre (teniendo en cuenta que el último semestre es de prácticas profesionales); se logró una muestra no probabilística de 105 estudiantes en los cuatro programas, distribuidos así: 26 estudiantes de la Universidad Francisco de Paula Santander, sede Cúcuta; 43 de la Universidad Francisco de Paula Santander, sede Ocaña; 18 estudiantes de la Universidad de Pamplona, sede Pamplona; y 18 de la Universidad de Pamplona, sede Villa del Rosario.

El instrumento fue aplicado en el primer semestre de 2016 y no se incluyeron los estudiantes de comunicación de la Universidad Libre sede Cúcuta y Universidad Minuto de Dios sede Cúcuta, porque sus estudiantes se encontraban cursando los primeros semestres del plan de estudios y no cumplían el criterio de selección. Respecto a la posibilidad de realizar un estudio comparativo de primeros semestres contra últimos semestres, en las etapas de diseño y validación del instrumento se consideró la población de primeros semestres pero fue descartada por el bajo o casi inexistente conocimiento sobre la temática. Lo anterior lleva a una primera conclusión: la mayoría de estudiantes que ingresan a los programas de comunicación no poseen un conocimiento previo sobre las aplicaciones tecnológicas que requiere la profesión.

Como herramienta de recolección de información, se diseñó una encuesta resultado de la revisión y análisis de 50 antecedentes, de los cuales destacan las investigaciones de Grandío-Pérez (2015), y Arras, Torres y García-Valcárcel (2011). Asimismo, se consultaron profesionales en comunicación y en estadística para validar teórica y cuantitativamente el instrumento.

La encuesta contó con cinco segmentos, los cuales se distribuyeron de la siguiente manera: (1) el uso que se les dan a diferentes plataformas web, (2) el nivel de dominio frente a herramientas de edición de audio, vídeo, imagen, texto, (3) razones para aprender o no sobre esta temática, (4) nivel de conocimiento sobre las funciones comunicativas de las plataformas web y (5) competencias éticas, investigativas y profesionales, asociadas al uso de las herramientas y plataformas digitales. Finalmente todas las encuestas fueron tabuladas y su análisis se presenta a continuación.

\section{Resultados y discusión}

Las herramientas digitales y plataformas web han logrado posicionarse como esenciales en el ejercicio de muchos profesionales debido a que facilitan el desarrollo de tareas académicas, laborales y sociales. 
La sociedad digital demanda, desde la concepción de Velandria y Villalobos (2008), que:

Las universidades deben considerar como prioritario la adquisición, el desarrollo y la gestión de su propia capacidad tecnológica, así lo afirma Villalobos (2004), quien apunta que hoy en día el concepto de analfabetismo no se limita al no saber leer, escribir y contar, ahora se refiere a la posibilidad de responder al desafío de la actualización tecnológica (p. 94).

Por tal motivo se indagó sobre el uso de herramientas y plataformas en estudiantes de Comunicación Social, considerando que en su vida laboral es indispensable el manejo de dichos entornos y herramientas. El primer segmento de preguntas abordó el uso que se da a las plataformas web; se encontró que -en promedioel $48 \%$ de los comunicadores en formación desconocen las plataformas para la creación de páginas web como Flickr, Pinterest, Wix y Jimdo, siendo Jimdo y Flickr las menos conocidas. El 32\% conoce pero no utiliza herramientas como Twitter, Flickr, Pinterest, Wix, Wordpress, Blogger y Jimdo. Sólo el 9\% indicó no saber usar la herramienta Pinterest y 7\% Flickr y Wordpress. Para fines sociales, las redes más usadas son Facebook con 81\%, Instagram con 79\%, YouTube con $65 \%$ y Twitter con $45 \%$.

\begin{tabular}{|l|c|c|c|c|c|c|c|c|c|c|}
\hline & Facebook & Twitter & Instagram & Youtube & Flickr & Pinterest & Wix & Wordpress & Blogger & Jimdo \\
\hline $\begin{array}{l}\text { NO LA } \\
\text { CONOZCO }\end{array}$ & $2 \%$ & $5 \%$ & $5 \%$ & $5 \%$ & $48 \%$ & $41 \%$ & $45 \%$ & $22 \%$ & $10 \%$ & $58 \%$ \\
\hline $\begin{array}{l}\text { LA CONOZCO } \\
\text { PERO NO LA } \\
\text { USO }\end{array}$ & $4 \%$ & $34 \%$ & $10 \%$ & $4 \%$ & $31 \%$ & $35 \%$ & $28 \%$ & $26 \%$ & $36 \%$ & $15 \%$ \\
\hline NO SÉ USARLA & $0 \%$ & $1 \%$ & $1 \%$ & $1 \%$ & $7 \%$ & $9 \%$ & $2 \%$ & $7 \%$ & $3 \%$ & $2 \%$ \\
\hline $\begin{array}{l}\text { LA USO CON } \\
\text { FINES SOCIALES }\end{array}$ & $81 \%$ & $45 \%$ & $79 \%$ & $65 \%$ & $6 \%$ & $9 \%$ & $2 \%$ & $2 \%$ & $6 \%$ & $3 \%$ \\
\hline $\begin{array}{l}\text { LA USO } \\
\text { CON FINES } \\
\text { ACADÉMICOS }\end{array}$ & $11 \%$ & $10 \%$ & $2 \%$ & $24 \%$ & $7 \%$ & $4 \%$ & $18 \%$ & $38 \%$ & $40 \%$ & $19 \%$ \\
\hline $\begin{array}{l}\text { LA USO } \\
\text { CON FINES } \\
\text { LABORALES }\end{array}$ & $2 \%$ & $5 \%$ & $4 \%$ & $2 \%$ & $2 \%$ & $3 \%$ & $6 \%$ & $6 \%$ & $5 \%$ & $3 \%$ \\
\hline \begin{tabular}{l} 
TOTAL \\
\hline
\end{tabular} & $100 \%$ & $100 \%$ & $100 \%$ & $100 \%$ & $100 \%$ & $100 \%$ & $100 \%$ & $100 \%$ & $100 \%$ & $100 \%$ \\
\hline
\end{tabular}

Tabla 1. Uso de la web 2.0 
Las redes sociales son utilizadas para fines de socialización en un 67\%, mientras que se utilizan en un 12\% para fines académicos y sólo en un 3\% para acciones laborales; con esos datos se puede inferir que, en su formación de pregrado, los estudiantes no usan estas herramientas o las emplean sólo para difundir información y no se perciben fines de comunicación estratégica.

Cabe resaltar que en la investigación realizada por Vidales (2012) en El Salvador, el uso de redes sociales con fines sociales se presenta como una realidad notoria; redes como Facebook -que plantean la interconexión global de personas- tienen predilección entre los jóvenes cuyas edades van de los “18 - 24 años” (p. 11), etapa de la vida en la que muchos de ellos se encuentran inmersos en un escenario universitario.

En Apartadó, Antioquia, también se encontró que: "los estudiantes valoran las posibilidades que ofrece el Internet, pero no lo utilizan para apoyar sus procesos de aprendizaje, lo hacen para chatear o comunicarse a través del correo, siendo estas opciones mínimas ofrecidas por la red" (Pineda \& García, 2010, p. 1). Este uso sería satisfactorio si no se tratara de profesionales en comunicación que requieren una visión estratégica de las herramientas digitales para su ejercicio profesional.

Al no explorar y emplear las herramientas para fines académicos, laborales y de comunicación estratégica, los comunicadores presentarán dificultades en su vida profesional al enfrentarse al reto de crear una marca de su propia identidad en entornos digitales como nueva forma de presentar su hoja de vida a posibles empleadores.

En general se evidencia un bajo nivel de conocimiento y uso de las herramientas señaladas, lo que contrasta con estudios como el de Marzal y Soler (2011), quienes encontraron un alto grado de uso de la plataforma Flickr por parte de los estudiantes de la Universidad Jaume I, facilitando el desarrollo de competencias en torno a la fotografía digital.

\begin{tabular}{|l|l|l|l|l|l|l|l|}
\hline & Corel & Photoshop & Illustrator & Lightroom & Pixelmator & Inkscape & Polarr \\
\hline $\begin{array}{l}\text { No sé } \\
\text { usarla }\end{array}$ & $14 \%$ & $16 \%$ & $43 \%$ & $80 \%$ & $84 \%$ & $88 \%$ & $88 \%$ \\
\hline Nivel Bajo & $13 \%$ & $22 \%$ & $18 \%$ & $10 \%$ & $10 \%$ & $10 \%$ & $10 \%$ \\
\hline $\begin{array}{l}\text { Nivel } \\
\text { medio }\end{array}$ & $32 \%$ & $33 \%$ & $20 \%$ & $7 \%$ & $5 \%$ & $2 \%$ & $2 \%$ \\
\hline Nivel alto & $32 \%$ & $20 \%$ & $17 \%$ & $3 \%$ & $1 \%$ & $0 \%$ & $1 \%$ \\
\hline Experto & $8 \%$ & $9 \%$ & $2 \%$ & $0 \%$ & $0 \%$ & $0 \%$ & $0 \%$ \\
\hline Total & $100 \%$ & $100 \%$ & $100 \%$ & $100 \%$ & $100 \%$ & $100 \%$ & $100 \%$ \\
\hline
\end{tabular}

Tabla 2. Herramientas para la edición de imágenes. 
Las herramientas de Corel, Photoshop, Illustrator, Ligthroom, Pixelmator, Inkscape, y Polarr, fueron escogidas como las más utilizadas por los profesionales en el área de comunicación. Sin embargo, los resultados en esta sección muestran que -en promedio- 58,9\% de los estudiantes indican no saber usar estas herramientas, sólo el 2,6\% dice haber logrado un dominio experto, de manera especial en Photoshop $(8,6 \%)$, Corel $(7,6 \%)$ e Illustrator $(1,9 \%)$.

Panorama preocupante en la era digital, que exige a los comunicadores tener nociones de diseño digital para desenvolverse en tareas que se relacionen de manera directa e indirecta en su oficio laboral. Desarrollar en un porcentaje mínimo el nivel de experticia en el manejo de herramientas que, según los profesionales consultados en el área de comunicación son indispensables, resulta una meta que desde la academia debe resaltarse. A continuación, en la Tabla 3, se presenta la consulta realizada frente al dominio de herramientas en torno a la edición de vídeo.

\begin{tabular}{|l|c|c|c|c|c|}
\hline & After Effects & Adobe Premier pro & Sony Vegas & Final Cut Pro & Movica \\
\hline No sé usarla & $58 \%$ & $35 \%$ & $51 \%$ & $68 \%$ & $79 \%$ \\
\hline Nivel Bajo & $14 \%$ & $12 \%$ & $16 \%$ & $11 \%$ & $12 \%$ \\
\hline Nivel medio & $23 \%$ & $22 \%$ & $9 \%$ & $8 \%$ & $5 \%$ \\
\hline Nivel alto & $4 \%$ & $21 \%$ & $17 \%$ & $11 \%$ & $1 \%$ \\
\hline Experto & $1 \%$ & $10 \%$ & $7 \%$ & $2 \%$ & $3 \%$ \\
\hline Total & $100 \%$ & $100 \%$ & $100 \%$ & $100 \%$ & $100 \%$ \\
\hline
\end{tabular}

Tabla 3. Herramientas para la edición de vídeo.

Los niveles alto y experto fueron indicados en promedio por el $13 \%$ de los estudiantes en herramientas como Aftter Efects, Adobe Premiere Pro, Sony Vegas, Final Cut y Movica, mientras que un 58\% no sabe usarlas y el 26\% se encuentra en un nivel medio y bajo en este segmento.

El mayor índice de experticia se encuentra en Adobe Premier Pro con un 9,5\%; la segunda herramienta con mayor índice de manejo es Sony Vegas con un 6,7\%. Hallazgo que, al igual que en la sección de herramientas de edición de imágenes digitales, presenta una estadística desfavorable porque más de la mitad de los estudiantes de diferentes instituciones de educación superior que ofertan el pregrado de Comunicación en Norte de Santander y que participaron de la presente investigación, no cuentan con las habilidades necesarias para la edición de vídeo. $\mathrm{Si}$ bien es cierto que en producción de televisión se cuenta con profesionales dedicados a la grabación y edición de vídeo y el profesional en comunicación puede no requerir dichas habilidades, cada día son más las empresas que buscan en el comunicador corporativo un periodista y realizador de productos audiovisuales, radiales y gráficos, que alimente sus redes sociales y páginas web. Un profesional integral que, de acuerdo a los resultados, no está siendo formado en la actualidad. 
Debe destacarse que el 84\% de los estudiantes no está desarrollando las competencias necesarias desde la academia, lo que plantea inquietudes sobre la apropiación de las TIC en el aula y si existen espacios en que se incentive en el estudiante su papel de emprendedor, es decir, su capacidad de aprendizaje autónomo para asegurar una actualización permanente en el uso de herramientas tecnológicas.

Si se considera que la encuesta se aplicó a estudiantes cuyo proceso de formación se encuentra a punto de culminar, es preocupante hallar que no cuentan con las habilidades necesarias en el componente tecnológico y que muchos de ellos pueden considerarse analfabetas digitales (Velandria y Villalobos, 2008).

\begin{tabular}{|l|c|c|c|c|}
\hline & Audacity & Sound Editor & Adobe Audition & Musical sketch pad \\
\hline No sé usarla & $58 \%$ & $57 \%$ & $20 \%$ & $75 \%$ \\
\hline Nivel Bajo & $13 \%$ & $16 \%$ & $7 \%$ & $15 \%$ \\
\hline Nivel medio & $13 \%$ & $13 \%$ & $16 \%$ & $7 \%$ \\
\hline Nivel alto & $7 \%$ & $10 \%$ & $31 \%$ & $1 \%$ \\
\hline Experto & $9 \%$ & $4 \%$ & $26 \%$ & $2 \%$ \\
\hline Total & $100 \%$ & $100 \%$ & $100 \%$ & $100 \%$ \\
\hline
\end{tabular}

Tabla 4. Dominio de herramientas de edición de audio.

Respecto a las herramientas de audio, los niveles alto y experto fueron alcanzados por un promedio de $22 \%$ de los estudiantes, frente al 53\% que no saben usar las herramientas básicas de edición de audio; en promedio, el 25\% se encuentra en niveles bajo y medio de uso. Se repite la tendencia de los editores audiovisuales -sean estos de edición de imágenes, vídeos o audios-: en términos generales, los estudiantes de comunicación social presentan falencias en competencias de edición.

Otras herramientas esenciales en la comunicación digital son las que permiten la creación de piezas gráficas dinámicas, como Prezi y Powtoon. 26\% de los estudiantes indicaron saber manejar Prezi como expertos y 14\% Powtoon. Queda la tarea tanto para estudiantes como para docentes- de promover el uso creativo de otras herramientas digitales en red y de trabajo colaborativo, para ser reproducido en redes de conocimiento, más allá del fin académico de la producción de contenidos para el aula.

Se indagó también por el uso de cámaras de producción visual o audiovisual. Resultados que se pueden observar en la siguiente tabla. 


\begin{tabular}{|l|c|c|c|}
\hline & Cámara fotográfica & Cámara de vídeo & Smartphone \\
\hline No sé usarla & $3 \%$ & $5 \%$ & $8 \%$ \\
\hline Nivel Bajo & $4 \%$ & $8 \%$ & $4 \%$ \\
\hline Nivel medio & $23 \%$ & $21 \%$ & $9 \%$ \\
\hline Nivel alto & $27 \%$ & $29 \%$ & $20 \%$ \\
\hline Experto & $44 \%$ & $38 \%$ & $60 \%$ \\
\hline Total & $100 \%$ & $100 \%$ & $100 \%$ \\
\hline
\end{tabular}

Tabla 5. Dominio en el uso de cámaras.

En esta sección de resultados se puede evidenciar un mayor dominio, si se compara con las demás herramientas. Al promediar los resultados, es de resaltar que sólo el 5\% de los estudiantes no sabe usar las cámaras de vídeo, de fotografía y el smartphone; $47 \%$ considera que las maneja en nivel experto y un $43 \%$ está en nivel medio y alto.

En el trabajo de aula en asignaturas de fotografía y producción de contenidos audiovisuales, se hace énfasis en el manejo del equipo y sus aspectos técnicos, y no tanto en herramientas de edición, como se pudo ver en segmentos anteriores. Se debe determinar si esta falencia en las competencias de edición corresponde a un sesgo del docente o a debilidades en la infraestructura y dotación en las universidades.

\section{1 ¿Qué causa el desuso de las TIC?}

Para profundizar en las razones que llevan a un estudiante a no reforzar sus saberes en el área de herramientas y plataformas digitales, se preguntó por las principales razones de tal conducta. El 34\% de los estudiantes dicen no tener acceso a las herramientas, $14 \%$ indica que no requieren de esta herramienta en su ejercicio académico/profesional, 38\% señala que falta mayor formación académica y un 14\% dice que no se encuentra interesado en el tema.

Quienes conocen y usan las herramientas, en un 15\% indican que lo hacen porque son autodidactas, otro $27 \%$ señalan que recibieron información por parte de la academia sobre la existencia y manejo básico de las mismas, el 20\% expresa un gusto en mantenerse actualizado en el uso de las herramientas comunicativas y un 38\% afirma que las emplea porque las requiere en su ejercicio académico/profesional. 
En este segmento resalta el hallazgo de un 38\% de estudiantes que indican falta de formación académica en las universidades. Al respecto, Guisao (2011) manifiesta que:

\footnotetext{
“(...) es crucial que en las instituciones de educación superior se de relevancia a la formación en el uso de las TIC y en competencias tecnológicas, puesto que son las indicadas para propiciar la apropiación, reflexión e interpretación de la cambiante sociedad del conocimiento" (p. 61).
}

Es evidente que las instituciones tienen un papel fundamental a la hora de señalar u orientar a los estudiantes en el conocimiento y uso de diferentes herramientas y plataformas que sean de utilidad para el desarrollo de su labor en lo académico y profesional.

En resumen, frente al dominio de las diferentes herramientas aquí reseñadas, se encontró que, en promedio, 44,3\% de los estudiantes encuestados indican no saber utilizar las herramientas, mientras solo el 15,4\% alcanzan un nivel de experticia. De tal manera que sólo ese 15\% - del total de los encuestados que pronto ejercerán su carrera en la región - se encuentran preparados para ello, mientras el otro 85\% deben reforzar sus habilidades para superar la brecha digital o verán disminuir sus oportunidades laborales al ser considerados analfabetas digitales.

\section{2 ¿Y para qué sirven las plataformas digitales?}

En esta sección se preguntó a los estudiantes por su conocimiento sobre las funciones comunicativas que poseen las plataformas digitales. Mediante una escala, primero se les consultó si conocían sus funciones, si sabían usarlas - es decir, si elaboraban productos comunicativos para tales plataformas- y, para finalizar, si contaban con conocimientos para evaluar el impacto de las estrategias comunicativas en plataformas digitales.

\begin{tabular}{|l|c|c|c|c|c|c|}
\hline & Facebook & Twitter & Instagram & Youtube & Flickr & Pinterest \\
\hline Desconozco sus funciones comunicativas & $6 \%$ & $15 \%$ & $9 \%$ & $7 \%$ & $61 \%$ & $62 \%$ \\
\hline $\begin{array}{l}\text { Identifico sus funciones comunicativas, } \\
\text { pero no se usarlas }\end{array}$ & $8 \%$ & $30 \%$ & $29 \%$ & $21 \%$ & $28 \%$ & $25 \%$ \\
\hline $\begin{array}{l}\text { Conozco y se usar algunas de sus } \\
\text { funciones comunicativas básicas }\end{array}$ & $47 \%$ & $31 \%$ & $34 \%$ & $33 \%$ & $6 \%$ & $6 \%$ \\
\hline $\begin{array}{l}\text { Domino la herramienta para la } \\
\text { elaboración de productos comunicativos }\end{array}$ & $29 \%$ & $19 \%$ & $26 \%$ & $31 \%$ & $6 \%$ & $7 \%$ \\
\hline $\begin{array}{l}\text { Domino su uso para evaluar el impacto de } \\
\text { las estrategias comunicativas }\end{array}$ & $11 \%$ & $4 \%$ & $3 \%$ & $8 \%$ & $0 \%$ & $1 \%$ \\
\hline & $100 \%$ & $100 \%$ & $100 \%$ & $100 \%$ & $100 \%$ & $100 \%$ \\
\hline
\end{tabular}

Tabla 6. Dominio de funciones comunicativas de las plataformas digitales. 
En primer lugar se halló que las redes sociales son las más conocidas, lo que no implica que se identifiquen claramente sus funciones comunicativas (como en el caso de Twitter e Instagram). Facebook y Youtube son las plataformas que los jóvenes más conocen y saben usar, pero en promedio sólo 30\% indica dominarlas para elaborar productos comunicativos. Cabe resaltar que en un momento anterior se reconoce que el $67 \%$ de los estudiantes se inclina por usar estas redes con fines sociales más que laborales.

Los resultados más desfavorables se encuentran en torno al quinto ítem, al preguntar a los estudiantes si dominan estas herramientas para evaluar el impacto de las estrategias comunicativas, sólo $11 \%$ de los estudiantes manifestó dominar su uso en el caso de Facebook, seguido por un 8\% que expresó dominar las herramientas de impacto y medición de YouTube. Se evidencian debilidades en la formación de pregrado en lo referente a big data y, en el caso específico de las redes, las funciones propias de medición y estadísticas de seguimiento de las publicaciones.

\subsection{Competencias éticas, investigativas y profesionales}

Las competencias son entendidas como los conocimientos, habilidades y valores que se requieren para desempeñarse en una ocupación; es posible observarlas y medirlas en función de saberes, como saber-conocer, saber-hacer y saber-ser.

Tobón, Rial, Carretero \& García (2006) indican que para definir las competencias se debe considerar los siguientes parámetros:

A) Articulación sistémica y en tejido de las actitudes, los conocimientos y las habilidades procedimentales; B) desempeño tanto ante actividades como con respecto al análisis y resolución de problemas; C) referencia a la idoneidad en el actuar. Las competencias son procesos complejos de desempeño con idoneidad en determinados contextos, teniendo como base la responsabilidad (p. 100).

En este caso se habla puntualmente de las competencias que debe desarrollar el comunicador social frente al uso de la tecnología desde aspectos éticos, investigativos y profesionales. Las competencias TIC deben entenderse como:

(...) habilidades, conocimientos y actitudes aplicadas a la utilización de sistemas de información y comunicación, así como el equipo que la actividad envuelve y, lo que deben saber y ser capaces de aprender y transferir de manera efectiva los estudiantes, con el fin de vivir productivamente en un mundo digital (Arras, Torres, GarcíaValcárcel y Muñoz-Repiso, 2011, p. 4).

A partir de Grandío-Pérez (2015) y Arras, Torres y García-Valcárcel (2011), se elaboró un listado de competencias ante las cuáles los estudiantes debían responder si las poseían o no. 


\begin{tabular}{|l|l|l|l|l|}
\hline & \multicolumn{1}{|c|}{} & SI & NO & TOTAL \\
\hline $\mathbf{1}$ & $\begin{array}{l}\text { Asumo una actitud responsable ante la propia identidad online/offline y de } \\
\text { los demás }\end{array}$ & $91 \%$ & $9 \%$ & $100 \%$ \\
\hline $\mathbf{2}$ & $\begin{array}{l}\text { Evalúo la calidad, pertinencia y veracidad de la información disponible en } \\
\text { internet }\end{array}$ & $83 \%$ & $17 \%$ & $100 \%$ \\
\hline $\mathbf{3}$ & $\begin{array}{l}\text { Analizo los riesgos y potencialidades de publicar y compartir información a } \\
\text { través de Internet. }\end{array}$ & $91 \%$ & $9 \%$ & $100 \%$ \\
\hline $\mathbf{4}$ & Promuevo comunicaciones asertivas y respetuosas en la web & $83 \%$ & $17 \%$ & $100 \%$ \\
\hline $\mathbf{5}$ & $\begin{array}{l}\text { Utilizo la información disponible en Internet con una actitud crítica y } \\
\text { reflexiva. }\end{array}$ & $88 \%$ & $12 \%$ & $100 \%$ \\
\hline $\mathbf{6}$ & $\begin{array}{l}\text { Aplico las normas de propiedad intelectual y licenciamiento existentes, } \\
\text { referentes al uso de información ajena y propia. }\end{array}$ & $78 \%$ & $22 \%$ & $100 \%$ \\
\hline
\end{tabular}

Tabla 7. Competencias éticas.

Los futuros comunicadores sociales manifiestan como principal debilidad en su formación ética el manejo de información con relación a los derechos de autor y propiedad intelectual, lo que coincide con los problemas evidenciados alrededor del mundo con las normas de citación de textos y la originalidad en la producción de contenidos.

El 90,8\% analiza los riesgos y potencialidades de publicar y compartir contenidos a través de Internet y un $87,8 \%$ utiliza la información de manera crítica y responsable, aunque presentan debilidades en la evaluación de la calidad, pertinencia y veracidad de sus publicaciones, lo que lleva al riesgo de compartir información poco confiable. En el caso de un comunicador en formación, es de particular importancia la verificación y contraste de las fuentes para garantizar la transmisión correcta del mensaje que quiere compartir.

Pese a que el $90 \%$ de los encuestados manifiesta ser responsable frente al manejo de su identidad on line y off line, resulta contradictorio que un $17 \%$ señale no promover comunicaciones asertivas y respetuosas en la web. Aquí se reitera un hallazgo anterior y es que, en su mayoría, los comunicadores no emplean sus redes sociales como espacios para crear una identidad propia como marca. 


\begin{tabular}{|c|c|c|c|c|}
\hline & & SI & NO & TOTAL \\
\hline 1 & $\begin{array}{l}\text { Sé buscar, ordenar, filtrar, conectar y analizar información disponible en Internet de } \\
\text { fuentes confiables e idóneas. }\end{array}$ & $78 \%$ & $22 \%$ & $100 \%$ \\
\hline 2 & $\begin{array}{l}\text { Identifico redes, bases de datos y fuentes de información que facilitan mis procesos } \\
\text { de investigación. }\end{array}$ & $83 \%$ & $17 \%$ & $100 \%$ \\
\hline 3 & $\begin{array}{l}\text { Utilizo redes profesionales y plataformas especializadas en el desarrollo de mis } \\
\text { investigaciones. }\end{array}$ & $63 \%$ & $37 \%$ & $100 \%$ \\
\hline 4 & $\begin{array}{l}\text { Represento e interpreto datos e información de mis investigaciones en diversos } \\
\text { formatos digitales. }\end{array}$ & $65 \%$ & $35 \%$ & $100 \%$ \\
\hline 5 & Contrasto y analizo información proveniente de múltiples fuentes digitales. & $73 \%$ & $27 \%$ & $100 \%$ \\
\hline 6 & $\begin{array}{l}\text { Divulgo los resultados de mis investigaciones utilizando las herramientas que me } \\
\text { ofrecen las TIC. }\end{array}$ & $54 \%$ & $46 \%$ & $100 \%$ \\
\hline 7 & $\begin{array}{l}\text { Participo activamente en redes y comunidades de práctica, para la construcción } \\
\text { colectiva de conocimientos con el apoyo de TIC. }\end{array}$ & $36 \%$ & $64 \%$ & $100 \%$ \\
\hline
\end{tabular}

Tabla 8. Competencias investigativas.

En las competencias investigativas se evidencian altos porcentajes de no cumplimiento: el 64\% de los estudiantes no participa en redes y comunidades para la construcción colectiva de conocimientos apoyados en TIC y un 46\% no divulga los resultados de sus investigaciones, es decir, deja de contribuir al aprendizaje colaborativo y no aporta materiales a las bases de datos y de recursos de aprendizaje.

Las fortalezas en esta área se encuentran en que el 83\% conoce las redes, bases de datos y fuentes de información necesarias para la investigación, saben buscar, ordenar, filtrar y conectar, entre otras; el 78\% analiza de fuentes confiables e idóneas la información disponible en Internet y el 73\% contrasta y analiza la información proveniente de múltiples fuentes digitales.

Todas estas son competencias básicas para desarrollar la investigación formativa o de aula, necesaria para las labores propias del aprendizaje; sin embargo, aquellas competencias propias de la producción investigativa, como utilizar redes profesionales y plataformas especializadas en el desarrollo de investigaciones (37\%) y representar e interpretar datos e información de investigaciones en diversos formatos digitales (35\%), tienen altos porcentajes de desuso.

En este segmento es evidente la falta de estímulos desde la academia para el desarrollo de las labores propias de la investigación formativa, que los estudiantes conozcan el campo y sean productivos en la construcción de nuevo conocimiento. 


\begin{tabular}{|c|c|c|c|c|}
\hline & & SI & NO & TOTAL \\
\hline 1 & Comprendo relatos diseñados para múltiples plataformas y su funcionalidad. & $62 \%$ & $38 \%$ & $100 \%$ \\
\hline 2 & Creo relatos multimodales para escenarios virtuales. & $36 \%$ & $64 \%$ & $100 \%$ \\
\hline 3 & Entiendo e interpreto lenguajes como icónico, sonoro, audiovisual y escrito. & $92 \%$ & $8 \%$ & $100 \%$ \\
\hline 4 & Identifico las particularidades expresivas de las plataformas propias de la web 2.0 & $85 \%$ & $15 \%$ & $100 \%$ \\
\hline 5 & $\begin{array}{l}\text { Comprendo el lenguaje hipertextual (saltar de un texto a otro del mismo código) e } \\
\text { hipermedial (conectar códigos diferentes). }\end{array}$ & $59 \%$ & $41 \%$ & $100 \%$ \\
\hline 6 & $\begin{array}{l}\text { Utilizo de manera combinada lenguajes como el icónico, sonoro, audiovisual y escrito, así } \\
\text { como las plataformas propias de la web móvil } 2.0\end{array}$ & $86 \%$ & $14 \%$ & $100 \%$ \\
\hline 7 & Construyo relatos a través un lenguaje hipertextual e hipermedial. & $54 \%$ & $46 \%$ & $100 \%$ \\
\hline 8 & Relaciono las producciones mediáticas con otras manifestaciones artísticas. & $58 \%$ & $42 \%$ & $100 \%$ \\
\hline 9 & $\begin{array}{l}\text { Transformo producciones artísticas, potenciando la innovación, la experimentación y la } \\
\text { sensibilización estética. }\end{array}$ & $56 \%$ & $44 \%$ & $100 \%$ \\
\hline
\end{tabular}

Tabla 9. Competencias profesionales.

En las competencias profesionales se demuestra un alto conocimiento, implementación y combinación de lenguajes icónicos, sonoros, audiovisuales y escritos propios de la web 2.0, pues en los apartados 3 y 6 , los porcentajes llegaron a $92 \%$ y $86 \%$ respectivamente. No obstante, se observa un contraste en la competencia 2 relacionada con la creación de relatos multimodales para los escenarios virtuales, que obtiene una respuesta negativa del 64\% e indica que, si bien los estudiantes saben elaborar productos para medios radiales, audiovisuales y escritos, entre otros, no necesariamente comprenden cómo integrarlos a escenarios multimedia donde convergen los medios.

Las demás competencias se hallan en un nivel medio con un promedio del 65\%, resultado coherente con los demás segmentos de la encuesta donde se han demostrado las debilidades, tanto en el manejo de herramientas de edición de productos comunicativos, como plataformas web para diseño de estrategias comunicativas.

\section{Conclusiones}

Los estudiantes de comunicación social poseen algunas nociones sobre el uso de herramientas digitales y plataformas - entre otros-, pero para usos sociales y de entretenimiento. Lo que respecta a sus usos laborales en el campo de la producción mediática y comunicación digital, presentan bajos niveles de uso y conocimiento de las herramientas tecnológicas a su disposición. Su desinterés sobre el tema evidencia que aún no perciben la web como un escenario laboral donde pueden desarrollar un perfil y una marca o identidad propia que sea su tarjeta de presentación. 
Las fortalezas de estos futuros profesionales están en el manejo de equipos de grabación y fotografía, pero al pasar a la edición quedan expuestas sus debilidades en el uso de programas de edición de audio, vídeo, gráficos y fotografía, así como la creación de páginas web, blogs y piezas gráficas como infografías. Se debe indagar si estas debilidades están relacionadas con la apatía del estudiante, son debilidades en la planeación curricular o responden a carencias en la infraestructura y dotación de las universidades que permita aprender-haciendo, en lo que concierne a la producción de piezas comunicativas para múltiples formatos y plataformas.

Se corroboró - en al menos el 28\% de los estudiantes- el desinterés por el tema y la falta de información sobre su importancia en el ejercicio académico/profesional. Por eso se recomienda a las universidades incluir más espacios de práctica en su planeación curricular, con ejercicios que se realicen de forma paralela al aprendizaje de la producción mediática y que permitan al estudiante conocer la realidad a la que se enfrentará y las habilidades específicas que demanda el mercado laboral.

Otra debilidad está en que no existe una cultura de aprendizaje autónomo, lo que es indispensable en el campo de la innovación tecnológica, un campo en el que se requiere actualización permanente. En este sentido, las universidades podrían realizar ofertas de educación continuada que salden estos vacíos en los profesionales.

Se halló un panorama preocupante: el 34\% de estudiantes indica no tener acceso a las herramientas, de tal manera que se encuentran limitados en el desarrollo de competencias TIC, pues al no contar con los recursos necesarios se limitan sus posibilidades de aprendizaje y se afectan, especialmente, aquellos con bajos recursos que no pueden comprar sus propios equipos y aprender desde casa.

El principal reto para la academia es promover una cultura de actualización y afianzamiento permanente para el logro del aprendizaje autónomo de sus estudiantes, al tiempo que les faciliten su inmersión en escenarios digitales. En cuanto a las competencias éticas, investigativas y profesionales, los futuros egresados de los programas de comunicación presentan debilidades en temas como propiedad intelectual y derechos de autor, la participación en comunidades científicas y el desarrollo y divulgación de resultados de proyectos de investigación, así como en la producción y difusión del conocimiento a través del uso de la tecnología.

En ese sentido, Arras, Torres y García - Valcárcel (2011), reconocen que el panorama aún es difuso en torno a la capacitación, optimización y desarrollo de habilidades digitales que sean idóneas para el panorama laboral. 


\section{Notas}

1 Comunicadora Social, Magister en Estudios Sociales y Culturales, Especialista en Práctica Pedagógica Universitaria, Especialista en Administración de la Tecnología Educativa.

${ }^{2}$ Estudiante de Comunicación Social en la Universidad Francisco de Paula Santander, Cúcuta.

${ }^{3}$ Estudiante de Comunicación Social en la Universidad Francisco de Paula Santander, Cúcuta.

\section{Referencias}

Arras Vota, A M d G; Torres Gastelú, C A; García-Valcárcel Muñoz-Repiso, A; (2011). Competencias en Tecnologías de Información y Comunicación (TIC) de los estudiantes universitarios. Revista Latina de Comunicación Social, () 1-26. Recuperado de http://www.redalyc.org/articulo. oa?id $=81921340018$

Grandío-Perez, M. (2015). Indicadores para la evaluación de la alfabetización transmedia en los estudios universitarios de Comunicación. En: en Formación, perfil profesional y consumo de medios de alumnos en Comunicación, Fernando Peinado Miguel (Coord.). Cuadernos Artesanos de Comunicación, 77. La Laguna (Tenerife): Latina. Url: http://www.revistalatinacs. org/067/cuadernos/2015/cac77.pdf; DOI: 10.4185/cac77

Guisao, G. (2011). Percepción de docentes y estudiantes en relación con el uso de las TIC en los procesos de enseñanza-aprendizaje. Obtenido de: http://repository.udem.edu.co/bitstream/ handle/11407/84/CD-ROM\%205933\%202011.pdf?sequence=1

Marzal, J. \& Soler, M; (2011). Hábitos de consumo y usos de la fotografía en la era digital entre estudiantes de Comunicación. Comunicar, XIX() 109-116. Recuperado de http://4www. redalyc.org/articulo.oa?id=15820024013

Pineda, D, \& García, A. (2010). La interacción en los procesos educativos, una didáctica para el aprendizaje autónomo de los estudiantes de grado 4 y 5 de las instituciones educativas Colmenas y Campoalegrito, pertenecientes al núcleo educativo Fermín López de la vereda Santa Bárbara. Colombia. [Proyecto de grado] Universidad Tecnológica de Pereira, Licenciatura En Comunicación e Informática Educativas. Obtenido de: http:/ / repositorio. utp.edu.co/dspace/bitstream/handle/11059/2091/3713P649.pdf;jsessionid=0049B19A EB639F5B0BC6BD1C3E0DBDFD? sequence $=1$

Tobón, S., Rial, A., Carretero, M. \& García, J. (2006). Competencias, calidad y Educación Superior. Bogotá, Colombia: Alma Mater Magisterio.

Velandria Ch., C. T. \& Villalobos G. F. (2008). La generación de competencias tecnológicas en la formación de comunicadores sociales. Quórum Académico, 5() 91-110. Recuperado de http://www. redalyc.org/articulo.oa?id=199016810005

Vidales, M. (2012). La relación entre jóvenes y TIC en la investigación en comunicación en El Salvador. Razón y Palabra, 17 Recuperado de http: / / www.redalyc.org/articulo.oa?id=199524700029

Recibido: 11 de enero de 2018 / Aprobado: junio 10 de 2018 